\title{
Two-month acute microangiopathic brain injury follow-up in severe COVID-19: a case report
}

\author{
Valeria Mundula1 ${ }^{1}$ Anna Stainer ${ }^{2}$, Francesca Motta', Michele Ciccarelli² \\ 'Department of Biomedical Sciences, Humanitas University, Pieve Emanuele, Milan 20090, Italy. \\ ${ }^{2}$ Respiratory Department, Humanitas Clinical and Research Center, IRCCS, Rozzano, Milan 20089, Italy.
}

Correspondence to: Dr. Valeria Mundula, Emergency Medicine Department, Humanitas Clinical and Research Center, IRCCS, via Manzoni 56, Rozzano, Milan 20089, Italy. E-mail: valeria.mundula@gmail.com

How to cite this article: Mundula V, Stainer A, Motta F, Ciccarelli M. Two-month acute microangiopathic brain injury follow-up in severe COVID-19: a case report. Neuroimmuno/ Neuroinflammation 2021;8:203-10.

http://dx.doi.org/10.20517/2347-8659.2020.41

Received: 20 May 2020 First Decision: 7 Jul 2020 Revised: 20 Jul 2020 Accepted: 30 Jul 2020 Available online: 21 Sep 2021

Academic Editor: Athanassios P. Kyritsis Copy Editor: Cai-Hong Wang Production Editor: Jing Yu

\begin{abstract}
Coronavirus disease-19 (COVID-19) is caused by a severe acute respiratory syndrome coronavirus-2 and was declared a pandemic in March 2020. It mainly causes upper respiratory symptoms, but an interstitial viral pneumonia may occur, in severe cases complicated by acute respiratory distress syndrome. Neurological involvement has been reported but has not been well investigated. A 75-year old man presenting with severe COVID-19 related pneumonia developed a severe cognitive impairment and a right temporal hemianopsia, with focal microangiopathy and subacute ischemic alterations detected on brain imaging, interpreted as vasculiticinflammatory injury. The neurological disorder was diagnosed only after he was extubated. A rehabilitation program was set up, so the patient had a complete cognitive recovery. Our case underlines how COVID-19 can lead to severe neurological sequelae, so neurological examination should be promptly performed when patients display signs of nervous system involvement, in order to prevent further damages.
\end{abstract}

Keywords: Severe acute respiratory syndrome coronavirus-2, coronavirus disease-19, neurological involvement, focal microangiopathy, vasculitic-inflammatory brain injury

\section{INTRODUCTION}

In December 2019, several cases of pneumonia of unknown origin were reported in Wuhan, Hubei province of the Republic of China. An emerging coronavirus, named novel coronavirus 2019 (2019-CoV)

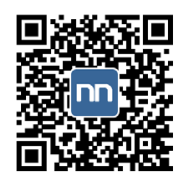


was then isolated on 7th January 2020 by the China Center for Disease Control (CDC) and lately renamed severe acute respiratory syndrome coronavirus-2 (SARS-CoV-2) by the World Health Organization (WHO). Infection caused by SARS-CoV-2 was first declared an outbreak by the WHO on the 30th of January 2020 and later a pandemic on the 11 th of March 2020.

Patients affected by coronavirus disease-2019 (COVID-19), the infection caused by SARS-CoV-2, mainly present with fever, cough, fatigue, dyspnea, constitutional symptoms such as headache or myalgia and gastrointestinal symptoms such as vomiting, diarrhea and nausea. More severe cases can develop viral pneumonia, acute respiratory distress syndrome, multiorgan failure and death ${ }^{[1]}$.

Coronaviruses have shown neurotropic and neuroinvasive capabilities and coronaviruses infections in humans have been associated with neurological manifestations ${ }^{[2]}$ such as seizures, change in mental status and encephalitis. Neurological involvement in COVID-19 has also been reported, particularly in severely ill patients, although evidence is still scarce ${ }^{[3]}$.

Here we describe a case of central nervous system (CNS) involvement in a patient affected by severe COVID-19.

\section{CASE REPORT}

A 75-year-old Caucasian male was referred to the Emergency Department of our hospital on 22nd March 2020 for dyspnea and fever in the previous week. His past medical history was consistent with systemic hypertension, well controlled with angiotensin receptor blocker and calcium channel blocker. No history of minor stroke or brain damage was found, no neurological investigation has ever been necessary during patient's life. He reported no allergies and he was an ex-smoker. The patient was referred to the emergency room where nasal swab for SARS-CoV-2 (SARS-CoV-2 Assay Allplex ${ }^{\mathrm{TM}}$ ) was performed and turned out positive. Arterial blood gas analysis revealed mild hypoxemic respiratory failure and a chest computed tomography scan showed bilateral ground-glass opacities [Figure 1].

Neurological examination was normal. The patient was diagnosed with COVID-19 pneumonia and was admitted to the medical ward. Low flow oxygen and antibiotic therapy with ceftriaxone were started. According to the Italian Society of Tropical and Infectious Diseases guidelines ${ }^{[4]}$ and based on the therapeutic evidences in March $2020^{[5]}$, hydroxychloroquine $200 \mathrm{mg}$ bid for ten days was administered and then prophylactic low molecular weight heparin (LMWH) was given, but no antiretroviral drugs were administered due to the presence of first degree atrioventricular block detected on an electrocardiogram.

Due to worsening respiratory conditions characterized by severe hypoxemic respiratory failure $\left(\mathrm{PaO}_{2} / \mathrm{FiO} 2\right.$ ratio 83), the patient was transferred to the intensive care unit (ICU) and invasive mechanical ventilation was initiated on March 24th. His blood exams are reported in Table 1.

Following intubation, a marked elevation of D-dimer ( $389 \mathrm{ng} / \mathrm{mL}$ to $39.000 \mathrm{ng} / \mathrm{mL}$ ), fibrinogen $(706 \mathrm{mg} / \mathrm{dL}$ ) and interleukin 6 (IL-6) (up to $136 \mathrm{pg} / \mathrm{mL}$, normal value $<40 \mathrm{pg} / \mathrm{mL}$ ) were noticed. Deep vein thrombosis and pulmonary embolism were excluded. In consideration of the new concern on COVID-19 derived prothrombotic state and microangiopathy ${ }^{[6,7]}$, LMWH was increased to anticoagulant therapeutic dosage (from March 28th to April 10th).

The patient stayed in the ICU for 17 days (where he was on propofol, rocuronium, fentanyl and noradrenaline), was extubated on the 8th of April and then was transferred to the clinical ward. No significant complications were reported except for Enterococcus faecium positivity on blood samples, not associated with a significant increase of inflammatory markers. However, vancomycin therapy was administered successfully. 


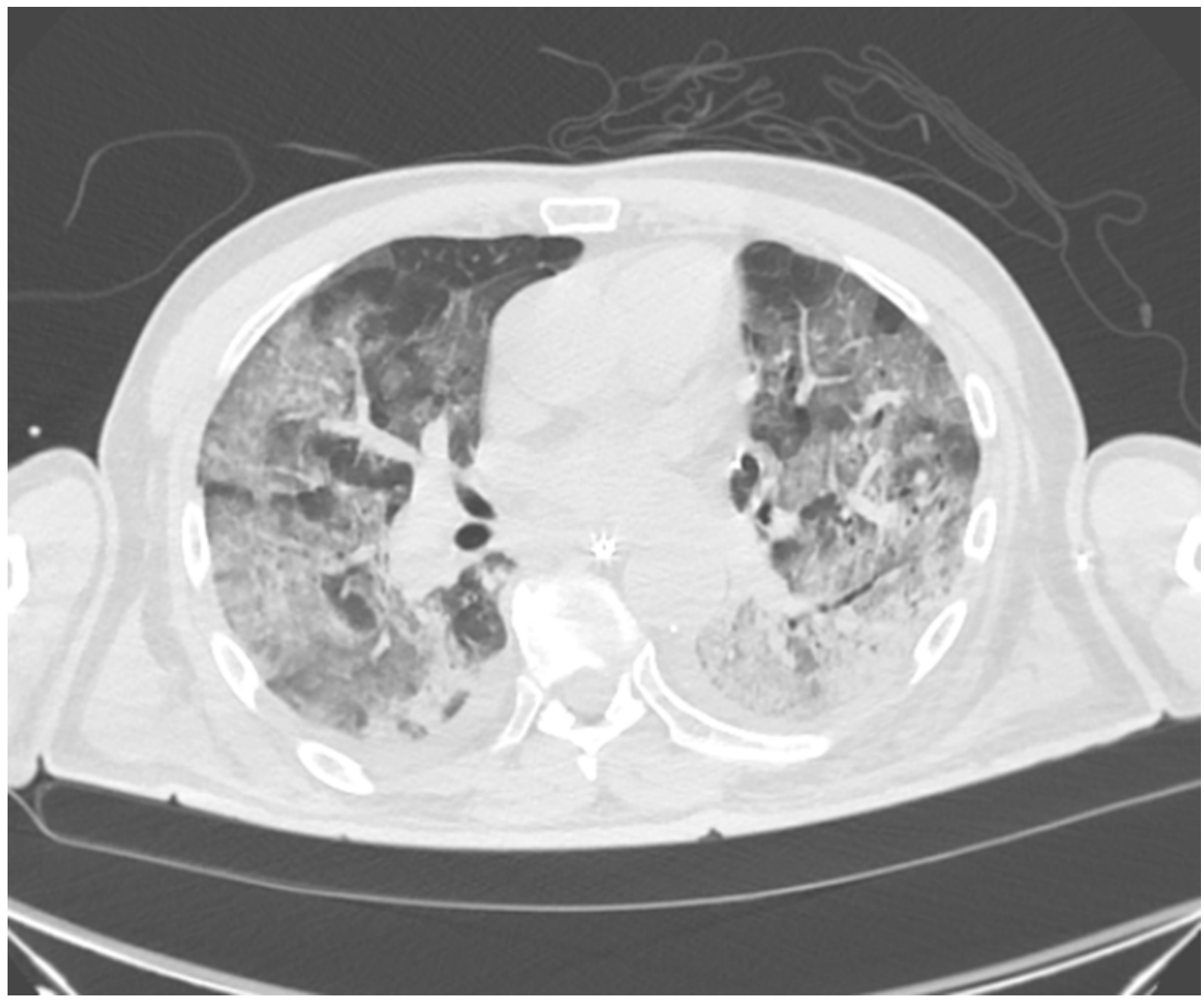

Figure 1. Chest computed tomography scan of patient performed on March 22nd showing diffuse ground-glass areas and lower lobes consolidations

\section{Table 1. Patient's blood exams}

\begin{tabular}{lllllll}
\hline Exams & Laboratory reference values & Admission & Intubation & Weaning & Ward & Recovery \\
\hline WBC $\left(10^{3} / \mathrm{mm}^{3}\right)$ & $4-10$ & 7.08 & 8.36 & 15.33 & 12.12 & 5.98 \\
Lymphocytes count & $1-4$ & 0.7 & 0.7 & 1.3 & 1.7 & 2 \\
$\left(10^{3} / \mathrm{mm}^{3}\right)$ & & & & & \\
$\left(10^{3} / \mathrm{mm}^{3}\right)$ & & 155 & 200 & 526 & 477 & 233 \\
PLT $\left(10^{3} / \mathrm{mm}^{3}\right)$ & $150-400$ & 1,910 & 2,321 & 870.3 & 697.7 & 456.8 \\
Ferritin $(\mathrm{ng} / \mathrm{mL})$ & $23-336$ & 599 & 657 & 477 & 369 & 177 \\
LDH $(\mathrm{IU} / \mathrm{L})$ & $<248$ & 1,344 & 975 & 458 & 157 & 20 \\
CPK (U/L) & $<172$ & 389 & 39,382 & 5,506 & 2679 & 518 \\
D-dimer $(\mathrm{ng} / \mathrm{mL})$ & $200-350$ & 21.39 & 33.03 & 17.36 & 4.63 & 0.77 \\
CRP $(\mathrm{mg} / \mathrm{dL})$ & $<0.5$ & 706 & 900 & 603 & 359 & 432 \\
Fibrinogen $(\mathrm{mg} / \mathrm{dL})$ & $160-400$ & $130 / 71$ & $141 / 90$ & $36 / 39$ & $48 / 46$ & $23 / 36$ \\
AST/ALT $(\mathrm{UI} / \mathrm{L})$ & $<51 /<51$ & $/$ & 136 & 49 & $/$ & $/$ \\
IL-6 $(\mathrm{pg} / \mathrm{mL})$ & $<40$ & & & &
\end{tabular}

WBC: white blood cells; PLT: platelets; LDH: lactate dehydrogenase; CPK: creatinine phosphokinase; CRP: C-reactive protein; AST/ALT: aspartate transaminase/alanine transaminase; IL-6: interleukine-6

After extubation, however, the patient showed neurological impairment. He was awake, but confused, agitated and uncooperating. Confabulation and echolalia were present. The patient only occasionally 

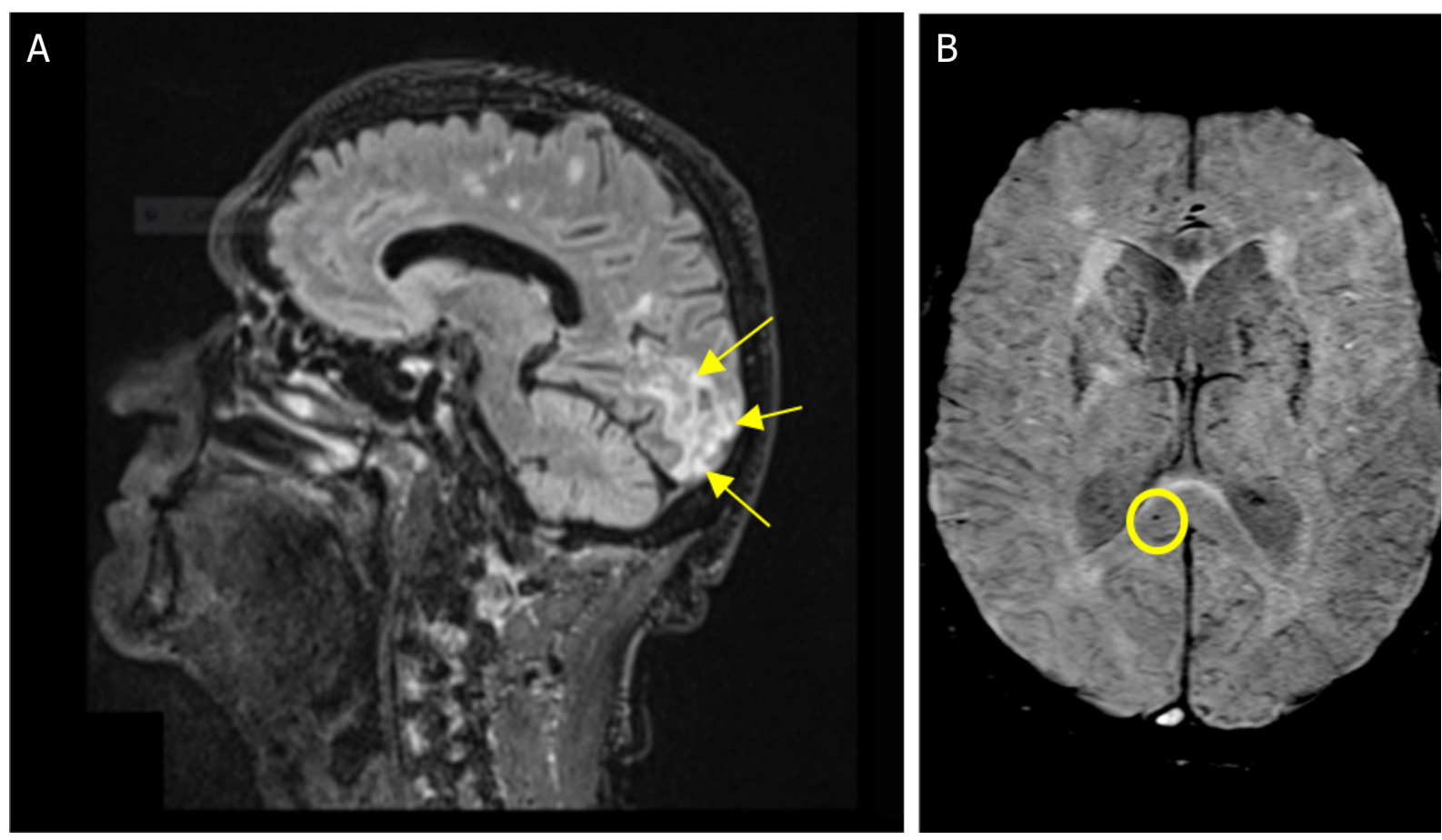

Figure 2. Magnetic resonance imaging fluid attenuated inversion recovery sagittal image showing cortical and subcortical ischemic lesions in occipital parasagittal areas (arrows), extending in calcarine fissure and superior lingual gyrus (A); susceptibility weighted imaging axial image showing some microbleeds in splenium of corpus callosus (circle) (B)

answered simple questions and rarely followed simple orders. He showed poor attention and concentration deficits. Moreover, he presented leg stiffness, but no rigor nucalis and no sensitivity nor motor alterations were noted. Grasping and suckling reflexes were detected, but neither Babinski reflex nor cranial nerve deficits were noted. Magnetic resonance imaging (MRI) of the head with gadolinium contrast and lumbar puncture for cerebrospinal fluid (CSF) analysis were performed. The MRI [Figure 2] excluded the presence of cerebral edema and major stroke. However, occipital lesions were detected, moreover, chronic vascular alteration with microhaemorragic foci were found, interpreted as focal microangiopathy with subacute ischemic lesions due to vasculitic-inflammatory injury.

Chemical-physical examination of CSF revealed clear fluid, containing $79 \mathrm{mg} / \mathrm{dL}$ glucose (reference interval 60-89 mg/dL), a slightly increased proteins level at $62.3 \mathrm{mg} / \mathrm{dL}$ (reference interval $15-45 \mathrm{mg} / \mathrm{dL}$ ) and no cells. Microbiological CSF analyses (bacteria, fungi, Herpes simplex-1 virus, Herpes simplex-6 virus and Varicella zoster virus) were negative. The patient was examined by a neurologist who attributed the neurological condition to a microthrombotic angiopathy in the context of a COVID-19 related altered immunity. Nonetheless, empiric antiviral therapy with acyclovir was started on April 17th for a duration of fifteen days, without significant improvement. A rehabilitation program was then started. We witnessed a slight and progressive neurological improvement of our patient's cognitive state, which allowed a gradual consensual improvement of his motor performance. He indeed became more cooperating and oriented both in the time and space, but still had episodes of confusion and confabulation. He developed anosognosia and intermittent episodes of coprolalia and palilalia. Stiffness improved such that, under medical supervision, the patient could assume the sitting position on the bed with good trunk control. Currently, the patient is awake, oriented both in space and time, cooperating, he answers simple and complex questions, follows simple and complex orders. He had a successful and complete cognitive recovery. In fact, he is able to do crosswords properly. He has no sensory nor motor alteration, he can perform postural steps completely unaided and he walks autonomously. A right temporal hemianopia has 
been observed as the patient became more cooperating, which did not improve during hospital stay. The patient will complete his rehabilitation program at home and a follow-up MRI with gadolinium contrast and a visual field exam have been scheduled.

\section{DISCUSSION}

Coronaviruses are primary respiratory viruses, causing mostly upper respiratory tract symptoms, but also bronchiolitis and pneumonia with dyspnea and respiratory distress, especially in elderly and frail patients $^{[8,9]}$. Nonetheless, coronaviruses capacity to invade the CNS has been described ${ }^{[10]}$. On December 2003 Hung et al. ${ }^{[11]}$ reported the first case of SARS-CoV CNS infection in a patient with SARS, with detection of viral RNA in CSF and blood samples. Desforges et al. ${ }^{[12]}$ well described the SARS-CoV neurotropism, highlighting that neuroinvasive coronaviruses can damage the CNS, leading to direct damage in the form of encephalitis, encephalopathy or indirect neurological disease, mediated by inflammation and microglia activation. Moreover, several studies demonstrated that human coronaviruses are structurally related to neuroinvasive animal coronaviruses ${ }^{[2]}$.

On March 2020 researchers from Beijing Ditan Hospital, China, described the first confirmed patient with COVID-19 whose CSF tested positive for SARS-CoV-2 RNA, after presenting with neurological disorders ${ }^{[13]}$. Other cases of neurologic alterations associated with COVID-19 were reported by Mao et al. ${ }^{[14]}$. Moreover, cases of encephalitis ${ }^{[15]}$, stroke ${ }^{[16]}$ or demyelinating lesions ${ }^{[17]}$ have been reported.

In our case we cannot exclude a direct viral cerebral injury, as we have not analyzed blood viral load by real time polymerase chain reaction (PCR), due to lack of equipment. It is possible that SARS-CoV-2 spread by hematogenous route into the CNS. A trans-neuronal route of neuroinvasion through the olfactory nerve is also possible, although less probable in our case, as the patient never suffered from anosmia. Cases of SARS-CoV-2 detection in CSF have been previously reported ${ }^{[18]}$. However, in several studies, CSF analysis was not performed, or when performed, SARS-CoV-2 was not detected ${ }^{[14,17,19-21]}$ despite a confirmed pneumonia caused by SARS-CoV-2 associated with onset of neurological symptoms.

We can also hypothesize that a cytokines release storm, by mechanism similar to that leading to lung damage $^{[22]}$, affected the brain. Two possible components could be involved. The first might have been the local hyperactivated immune response induced by SARS-CoV-2, as already described for other viruses ${ }^{[23]}$, leading to an intense inflammation of the brain parenchyma. The second might have been the activation of the endothelial cells due to cytokines and chemokines release, leading to a local pro-thrombotic state ${ }^{[24]}$. New evidences highlight hyperinflammation ${ }^{[25]}$ and thrombosis ${ }^{[6,7]}$ as important pathogenic mechanisms in COVID-19. The occurrence of both venous and arterial thrombosis in COVID-19 has been reported ${ }^{[6]}$. Moreover, capillary congestion, microthrombi and vasculitis have been detected post-mortem in lungs of a small cohort of deceased COVID-19 patients, despite anticoagulation ${ }^{[26]}$. A pro-thrombotic state with microthrombi development in the pulmonary circulation could hint the worsening of the ventilation/ perfusion ratio. The altered cross talk between inflammation, coagulation and the complement system could culminate in a generalized thrombotic state ${ }^{[20]}$, with organ failure and death ${ }^{[27]}$. Moreover, the endothelial role in brain damage is supported by the endothelial cells' high expression of ACE-2 receptors, which have been reported as a target of SARS-CoV-2 infection ${ }^{[28]}$. This binding may also lead to an inflammatory endothelial dysfunction, causing an alteration in brain autoregulation of cerebral circulation ${ }^{[29]}$.

In our case we suggest a multifactorial mechanism of brain damage perpetrated by SARS-CoV-2. All the described mechanisms, direct or indirect, might have had a role in the pathogenesis of the brain damage of the patient, also with different timing. In our patient, we could hypothesize that the virus disseminated via the bloodstream, possibly due to the host risk factors, such as old age and hypertension, leading to the first "hit" in the lung and brain parenchyma. The dysregulated and exuberant immune response, with the 
cytokines release storm, determined the second "hit", causing leukocytes recruitment and organ infiltration, endothelial activation ${ }^{[30]}$ and local microangiopathy ${ }^{[31]}$, responsible of further damage.

Of note, when the respiratory failure occurred, a proper neurological examination of our patient could not have been performed since he was urgently intubated. The observed increase of CRP, IL-6, D-dimer, and fibrinogen could have been markers of hyper-inflammation, prothrombotic state and endothelial activation. Ventilatory and neurological worsening probably occurred with a similar timing in the context of COVID-19 progression, because of what was previously described, but the neurological clinical features became apparent only after intubation weaning. Mao et al. ${ }^{[14]}$, in their multicentric observational study, pointed out how patients with neurological manifestations had more severe acute respiratory syndrome and higher D-dimer and fibrinogen levels than patients who did not develop neurological manifestations.

Our report has some limitations. PCR for SARS-CoV-2 was not performed on CSF, even though a negative result would not have excluded the presence of the virus in the $\mathrm{CNS}^{[21]}$. Of note, Li et al. ${ }^{[32]}$ described how virus particles were detected exclusively in neuronal cells. Moreover, several authors reported low sensibility of method ${ }^{[33-35]}$.

Electroencephalogram was not performed due to lack of instrumentation in COVID patients' ward, however the diagnostic specificity of this methodology is not always supported in patients with neurological manifestations in the context of COVID-19 ${ }^{[21]}$.

To our knowledge, no large cohort studies on brain MRI abnormalities in COVID-19 patients have been performed to date; Kremer et al. ${ }^{[21]}$ described eight brain MRI patterns, including FLAIR hyperintensities located in temporal lobe and hyperintense white matter, in a cohort of 37 patients, but, similarly to our case, the pathophysiological mechanism remained unclear. Moreover, only one patient was found positive on CSF PCR test for SARS-CoV-2 and EEG, when performed, showed nonspecific alterations in the $27 \%$ of cases.

Fitsiori et al. ${ }^{[36]}$ have recently described how patients who suffered from severe and moderate pulmonary COVID-19 requiring ICU mechanical ventilation developed neurological impairment. They pointed out that brain MRI showed multiple microbleeds involving atypical area, such as the corpus callosum, similarly to our case [Figure 2]. Anzalone et al. ${ }^{[20]}$ described MRI alterations in four cases of subacute encephalopathy in severe COVID-19 patients requiring intubation.

Our case demonstrates that patients with COVID-19 can have neurological manifestations and severe neurological sequelae. Rapid deterioration of respiratory conditions could lead to delayed diagnosis of neurological involvement. For this reason, we believe that neurological involvement should be investigated as soon as possible to ensure a greater possibility of diagnosis and neurorehabilitation. Moreover, the pathophysiological mechanism of CNS damage is still not completely understood. Neurological examination as well as CSF examination and radiologic studies should be performed in all patients displaying signs of CNS involvement, to allow a prompt intervention and prevent further damage.

\section{DECLARATIONS}

\section{Authors' contributions}

Planned and wrote the case report: Mundula V, Stainer A, Motta F

Collected the data: Mundula V

Supervised data entry and reviewed the manuscript: Stainer A, Motta F

Reviewed and revised the manuscript: Ciccarelli $\mathrm{M}$

Contributed and approved the submitted version of the manuscript: Mundula V, Stainer A, Motta F, Ciccarelli M 


\section{Availability of data and materials}

Not applicable.

\section{Financial support and sponsorship}

None.

\section{Conflicts of interest}

All authors declared that there are no conflicts of interest.

\section{Ethical approval and consent to participate}

Not applicable.

\section{Consent for publication}

Written informed consent for the publication of patients' information has been obtained from the patient.

\section{Copyright}

(c) The author(s) 2021.

\section{REFERENCES}

1. Kakodkar P, Kaka N, Baig M. A comprehensive literature review on the clinical presentation, and management of the pandemic coronavirus disease 2019 (COVID-19). Cureus 2020;12:e7560.

2. Desforges M, Le Coupanec A, Dubeau P, Bourgouin A, Lajoie L, et al. Human coronaviruses and other respiratory viruses: underestimated opportunistic pathogens of the central nervous system? Viruses 2019;12:14.

3. Asadi-Pooya AA, Simani L. Review, central nervous system manifestations of COVID-19: A systematic. J Neurol Sci 2020;413:116832.

4. SIMIT. Linee guida sulla gestione terapeutica e di supporto per pazienti con infezione da coronavirus COVID-19. Available from: https:// www.regione.lazio.it/binary/rl_sanita/tbl_contenuti/COVID19_LG_SIMITLombardia_2.0.pdf. [Last accessed on 4 Aug 2020]

5. Gao J, Tian Z, Yang X. Breakthrough: Chloroquine phosphate has shown apparent efficacy in treatment of COVID-19 associated pneumonia in clinical studies. Biosci Trends 2020;14:72-3.

6. Lodigiani C, Iapichino G, Carenzo L, Cecconi M, Ferrazzi P, et al; Humanitas COVID-19 Task Force. Venous and arterial thromboembolic complications in COVID-19 patients admitted to an academic hospital in Milan, Italy. Thromb Res 2020;191:9-14.

7. Magro C, Mulvey JJ, Berlin D, Nuovo G, Salvatore S, et al. Complement associated microvascular injury and thrombosis in the pathogenesis of severe COVID-19 infection: a report of five cases. Transl Res 2020;220:1-13.

8. Weiss SR, Navas-Martin S. Coronavirus pathogenesis and the emerging pathogen severe acute respiratory syndrome coronavirus. Microbiol Mol Biol Rev 2005;69:635-64.

9. Su S, Wong G, Shi W, Liu J, Lai ACK, et al. Epidemiology, genetic recombination, and pathogenesis of coronaviruses. Trends Microbiol 2016;24:490-502.

10. Li Z, Liu T, Yang N, Han D, Mi X, et al. Neurological manifestations of patients with COVID-19: potential routes of SARS-CoV-2 neuroinvasion from the periphery to the brain. Front Med 2020;4:1-9

11. Hung EC, Chim SS, Chan PK, Tong YK, Ng EK, et al. Detection of SARS coronavirus RNA in the cerebrospinal fluid of a patient with severe acute respiratory syndrome. Clin Chem 2003;49:2108-9.

12. Desforges M, Le Coupanec A, Stodola JK, Meessen-Pinard M, Talbot PJ. Human coronaviruses: viral and cellular factors involved in neuroinvasiveness and neuropathogenesis. Virus Res 2014;194:145-58.

13. Sun T, Guan J. Novel coronavirus and the central nervous system. Eur J Neurol 2020; doi: 10.1111/ene.14227.

14. Mao L, Jin H, Wang M, Hu Y, Chen S, et al. Neurologic manifestations of hospitalized patients with coronavirus disease 2019 in Wuhan, China. JAMA Neurol 2020:77:1-9.

15. Moriguchi T, Harii N, Goto J, Harada D, Sugawara H. A first case of meningitis/encephalitis associated with SARS-coronavirus-2. Int J Infect Dis 2020;94:55-8.

16. Morassi M, Bagatto D, Cobelli M, D’Agostini S, Gigli GL, et al. Stroke in patients with SARS-CoV-2 infection: case series. J Neurol 2020;267:2185-92.

17. Zanin L, Saraceno G, Panciani PP, Renisi G, Signorini L, et al. SARS-CoV-2 can induce brain and spine demyelinating lesions. Acta Neurochir 2020;162:1491-4

18. Zhang T, Rodricks MB, Hirsh E. COVID-19-associated acute disseminated encephalomyelitis: a case report. Available from: https://www. medrxiv.org/content/10.1101/2020.04.16.20068148v1. [Last accessed on 4 Aug 2020]

19. Bernard-Valnet R, Pizzarotti B, Anichini A, Demars Y, Russo E, et al. Two patients with acute meningo-encephalitis concomitant to SARS-CoV-2 infection. Eur J Neurol 2020; doi: 10.1111/ene.14298.

20. Anzalone N, Castellano A, Scotti R, Scandroglio AM, Filippi M, et al. Multifocal laminar cortical brain lesions: a consistent MRI finding 
in neuro-COVID-19 patients. J Neurol 2020:1-4.

21. Kremer S, Lersy F, de Sèze J, Ferré JC, Maamar A, et al. Brain MRI findings in severe COVID-19: a retrospective observational study. Radiology 2020;202222.

22. Pedersen SF, Ho YC. SARS-CoV-2: a storm is raging. J Clin Invest 2020;130:2202-5.

23. Schwerk C, Tenenbaum T, Kim KS, Schroten H. The choroid plexus-a multi-role player during infectious diseases of the CNS. Front Cell Neurosci 2015;9:80

24. Leisman DE, Deutschman CS, Legrand M. Facing COVID-19 in the ICU: vascular dysfunction, thrombosis, and dysregulated inflammation. Intensive Care Med 2020;46:1105-8.

25. Ceribelli A, Motta F, De Santis M, Ansari AA, Ridgway WM, et al. Recommendations for coronavirus infection in rheumatic diseases treated with biologic therapy. J Autoimmun 2020;109:102442.

26. Menter T, Haslbauer JD, Nienhold R, Savic S, Hopfer H, et al. Postmortem examination of COVID-19 patients reveals diffuse alveolar damage with severe capillary congestion and variegated findings in lungs and other organs suggesting vascular dysfunction. Histopathology 2020; doi: 10.1111/his.14134.

27. Henry BM, Vikse J, Benoit S, Favaloro EJ, Lippi G. Hyperinflammation and derangement of renin-angiotensin-aldosterone system in COVID-19: a novel hypothesis for clinically suspected hypercoagulopathy and microvascular immunothrombosis. Clin Chim Acta 2020;507:167-73.

28. Li H, Xue Q, Xu X. Involvement of the Nervous System in SARS-CoV-2 Infection. Neurotox Res 2020;38:1-7.

29. Wu Y, Xu X, Chen Z, Duan J, Hashimoto K, et al. Nervous system involvement after infection with COVID-19 and other coronaviruses. Brain Behav Immun 2020;87:18-22.

30. Varga Z, Flammer AJ, Steiger P, Haberecker M, Andermatt R, et al. Endothelial cell infection and endotheliitis in COVID-19. Lancet 2020;395:1417-8.

31. Mcgonagle D, O'donnell JS, Sharif K, Emery P, Bridgewood C. Immune mechanisms of pulmonary intravascular coagulopathy in COVID-19 pneumonia. Lancet Rheumatol 2020;2:e437-45.

32. Li Y, Bai W, Hashikawa T. The neuroinvasive potential of SARS-CoV2 may play a role in the respiratory failure of COVID-19 patients. J Med Virol 2020;92:552-5.

33. Helms J, Kremer S, Merdji H, Clere-Jehl R, Schenck M, et al. Neurologic features in severe SARS-CoV-2 infection. N Engl J Med 2020;382:2268-70.

34. Ye M, Ren Y, Lv T. Encephalitis as a clinical manifestation of COVID-19. Brain Behav Immun 2020:945-6.

35. Panciani PP, Saraceno G, Zanin L, Renisi G, Signorini L, et al. SARS-CoV-2: "Three-steps" infection model and CSF diagnostic implication. Brain Behav Immun 2020;87:128-9.

36. Fitsiori A, Pugin D, Thieffry C, Lalive P, Vargas MI. Unusual microbleeds in brain MRI of Covid-19 patients. J Neuroimaging 2020; doi: 10.1111/jon.12755. 